nephron

Practice
Nephron 2019;141:249-255

DOI: $10.1159 / 000495886$
Received: June 25, 2018

Accepted after revision: November 28, 2018

Published online: January 24, 2019

\title{
The Prevalence and Clinical Features of Fabry Disease in Hemodialysis Patients: Russian Nationwide Fabry Dialysis Screening Program
}

\author{
Sergey Moiseev ${ }^{a, c}$ Victor Fomin ${ }^{a} \quad$ Kirill Savostyanov ${ }^{b}$ Alexander Pushkov ${ }^{b}$ \\ Alexey Moiseev ${ }^{c}$ Andrey Svistunov ${ }^{a}$ Leyla Namazova-Baranova ${ }^{b}$

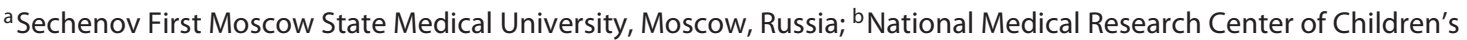 \\ Health, Moscow, Russia; ' ${ }^{C}$ Lomonosov Moscow State University, Moscow, Russia
}

\section{Keywords \\ Fabry disease $\cdot$ Screening $\cdot$ Dialysis}

\begin{abstract}
Aim: To evaluate the prevalence and clinical features of Fabry disease in patients with end-stage renal disease (ESRD) undergoing chronic hemodialysis.Methods: $a-G a l a c t o s i d a s e$ $A$ activity was measured in the dried blood spots by tandem mass spectrometry in 5,572 dialysis patients (63.7\% males). Diagnosis of Fabry disease was confirmed by sequencing of the GLA gene and by evaluating the globotriaosylsphingosine level in the dried blood spots. Results: Fabry disease was diagnosed in $20(0.36 \%)$ patients at the median age of 43 years $(28 ; 58)$. There were 19 males and 1 female. The prevalence of Fabry disease in dialysis patients was $0.53 \%$ in males and $0.05 \%$ in females. However, it was higher in males aged 30-49 years. Seventeen different GLA mutations were identified; 5 of them were novel. The median age at the initiation of hemodialysis was similar between patients with missense and nonsense mutations. Sixteen patients (80.0\%)
\end{abstract}

presented with typical symptoms of Fabry disease from childhood (neuropathic pain in 16, angiokeratoma in 7 and hypohidrosis/anhidrosis in 16). All patients had left ventricular hypertrophy, and 8 patients (40\%) had a history of ischemic stroke. Two patients died (recurrent stroke in one and sudden cardiac death in another patient). Conclusions: Screening in at-risk patients remains the feasible approach to diagnose Fabry disease in patients with ESRD and their family members, given a low awareness of Fabry disease among the Russian nephrologists. @ 2019 The Author(s)

Published by S. Karger AG, Basel

\section{Introduction}

Fabry disease is a rare $\mathrm{X}$-linked storage disease resulting from the deficient activity of the lysosomal a-galactosidase A (AGAL) and leading to a progressive accumulation of glycosphingolipids in a wide range of cell types throughout the body. Deposition of glycosphingo-

\begin{tabular}{|c|c|}
\hline KARGER & $\begin{array}{l}\text { (c) } 2019 \text { The Author(s) } \\
\text { Published by S. Karger AG, Basel }\end{array}$ \\
\hline $\begin{array}{l}\text { E-Mail karger@karger.com } \\
\text { www.karger.com/nef }\end{array}$ & $\begin{array}{l}\text { This article is licensed under the Creative Commons Attribution- } \\
\text { NonCommercial-NoDerivatives } 4.0 \text { International License (CC BY- } \\
\text { NC-ND) (http://www.karger.com/Services/OpenAccessLicense). } \\
\text { Usage and distribution for commercial purposes as well as any dis- } \\
\text { tribution of modified material requires written permission. }\end{array}$ \\
\hline
\end{tabular}

Sergey Moiseev, MD

Tareev Clinic of Internal Diseases

Sechenov First Moscow State Medical University

Rossolimo, 11/5, Moscow 119435 (Russia)

E-Mail clinpharm@mtu-net.ru 
lipids in podocytes, mesangium, glomerular endothelium, epithelium of the distal tubule, arterial and arteriolar endothelial and smooth muscle cells, and interstitial cells is associated with interstitial fibrosis and glomerulosclerosis and a progressive decline in kidney function. In patients with Fabry disease, the rate of occurrence and severity of nephropathy increase with age. Hemizygous males typically present with albuminuria and normal renal function in adolescence, develop overt proteinuria and progressive renal impairment by the second to fifth decades of life, and ultimately reach end-stage renal disease (ESRD) [1]. In untreated patients with Fabry disease, proteinuria was a major risk factor for the progression of kidney damage. In heterozygous females, the clinical expression of Fabry disease is more variable than in males. Heterozygous females are frequently asymptomatic or mildly symptomatic due to random $\mathrm{X}$-chromosome inactivation leading to a mosaic of cell populations. Skewed $\mathrm{X}$-chromosome inactivation can favor the expression of the mutant allele that is associated with the development of clinical symptoms of Fabry disease [2]. Therefore, female patients also exhibit signs of kidney damage, which is albuminuria/proteinuria and reduced estimated glomerular filtration rate, but at a lower percentage compared with males [3]. According to the Fabry Registry, $45 \%$ of males and $20 \%$ of females over 40 years of age had stage 3-5 chronic kidney disease (CKD) [4]. In the United States Renal Disease System database, women constituted only $12 \%$ of all patients with Fabry disease and ESRD [5].

Typical symptoms of Fabry disease including neuropathic pain, angiokeratoma, hypohidrosis/anhidrosis, and gastrointestinal disorders (nausea, vomiting, abdominal distension, episodic diarrhea, and constipation) are usually present from childhood or adolescence, and precede the development of renal, cardiac, and cerebrovascular diseases. However, the databases show a long delay between the onset of initial symptoms and diagnosis as a result of low awareness of the rare diseases [6]. Late diagnosis is also common in patients with a late-onset or attenuated phenotype of Fabry disease often affecting a single organ system, commonly the heart [7].

Undiagnosed patients with Fabry disease can be detected by screening in at-risk populations, such as patients with ESRD undergoing dialysis or renal transplantation, patients with hypertrophic cardiomyopathy or unexplained left ventricular hypertrophy, young adults (18-55 years) with a history of stroke or transient ischemic attack. Diagnostic performance of screening strategies requires further studies, since in one program chron- ic limb pain did not appear to be highly predictive of a diagnosis of Fabry disease in children and adolescents, suggesting that the key early signs and symptoms of Fabry disease are not specific to the disease [8].

In 2014-2016, a nationwide screening for Fabry disease among hemodialysis patients was conducted in Russia Fabry dialysis screening program. The aim of the current study was to evaluate the prevalence and clinical features of Fabry disease in patients with ESRD undergoing chronic hemodialysis.

\section{Materials and Methods}

A nationwide screening for Fabry disease among adult ( $\geq 18$ years) hemodialysis patients was performed in 157 units in different regions of Russia. All patients who participated in the Fabry dialysis screening program have provided an informed consent for screening test. There were no criteria of exclusion. However, patients with known FD were not tested.

\section{$\alpha$-Galactosidase A Activity}

AGAL activity was determined from filter paper dried blood spots by a validated ultra-performance liquid chromatography tandem mass spectrometry (Bruker Maxis Impact, Germany). Samples were taken on a dialysis day before the session. Substrates and internal standards were provided by the CDC (USA). The cutoff level of $>1.89 \mu \mathrm{mol} / \mathrm{L} / \mathrm{h}$ was determined as $10 \%$ of the mean control value (1,000 negative control samples).

\section{Genetic Testing}

Genetic testing was performed in patients with low AGAL activity. The coding exons (1-7) and flanking intronic regions of the GLA gene were amplified from purified genomic DNA by PCR. These purified DNA amplicons were sequenced with the Big Dye Terminator version 3.1 Cycle Sequencing Kit (Thermo Fisher Scientific). The products were purified with the Big Dye XTerminator Purification Kit (Thermo Fisher Scientific) and resolved using the ABI 3,500xL Genetic Analyzer. Data were analyzed using the ABI Data Collection software version 3.0, Sequencing Analysis software 5.2, and SeqScape software version 2.6. Sequences were compared to the reference DNA sequence (GenBank Accession: NM_000169.2).

\section{Diagnostic Criteria}

Criteria for a definite diagnosis of Fabry disease included a decreased or absent AGAL activity ( $<5 \%$ from the mean control value) and mutation in the GLA gene combined with at least one specific symptom (neuropathic pain, cornea verticillata, angiokeratoma), and/or increased globotriaosylsphingosine (Lyso-GL3), and/or an affected family member with a definite diagnosis. Fabry neuropathic pain was defined as pain in hands and/or feet with an onset of pain in childhood or adolescence, and/or a course characterized by exacerbations that are provoked by fever, exercise or heat. Angiokeratoma met the clinical criteria if they were clustered and present in characteristic areas: bathing trunk area, lips, and umbilicus. Cornea verticillata is a whorl-like pattern of corneal 
Table 1. Clinical and demographic characteristic of patients

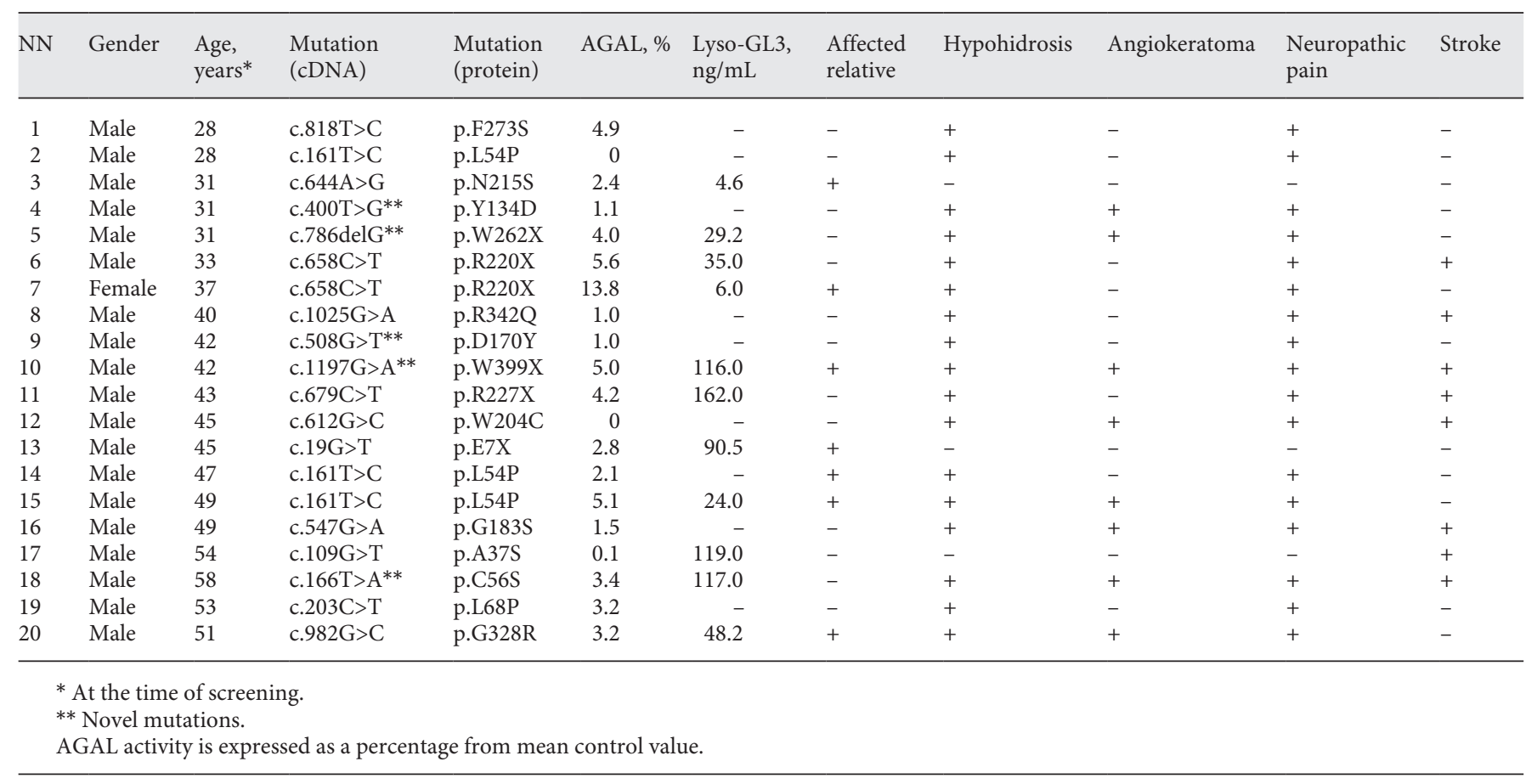

opacities in the absence of amphiphilic drug use (i.e., amiodarone, chloroquine) [9]. Lyso-GL3 was measured by tandem mass spectrometry in dried blood spots in the laboratories of Centogene AG (Rostock, Germany) or ARCHIMED Life Science GmbH (Vienna, Austria). The cut-off values were $\leq 1.8$ and $0.0-3.5 \mathrm{ng} / \mathrm{mL}$, respectively.

Chloroquine-induced phospholipidosis was excluded in the newly diagnosed Fabry patients.

\section{Statistical Methods}

Statistical hypothesis was not tested. Continuous data are shown as medians. Categorical data are given as absolute counts and as proportions. Medians were compared using Wilcoxon test. Statistical analysis was conducted using IBM SPSS Statistics 22.

\section{Results}

\section{Prevalence of Fabry Disease in Dialysis Patients}

AGAL activity was measured in the dried blood spots in 5,572 patients with ESRD undergoing hemodialysis. There were 3,551 males and 2,021 females. Fabry disease was diagnosed in 20 patients $(0.36 \%, 95 \%$ CI $0.20-0.52)$ at the median age of 43 years (28-58 years), including 19 males and 1 female. The latter was a 38-year-old female with a history of neuropathic pain and hypohidrosis, nonsense mutation in the GLA gene (c.658C > T), moder-

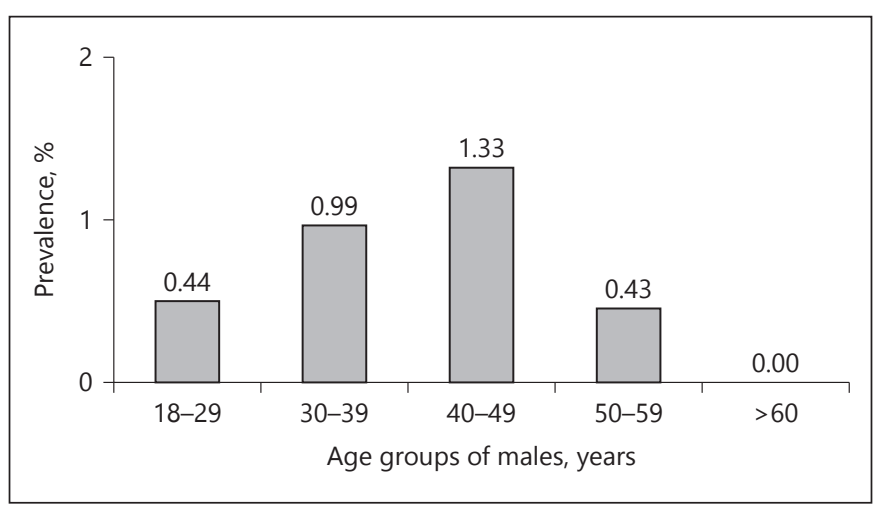

Fig. 1. Prevalence of Fabry disease in males of different age.

ately reduced AGAL activity, and increased lyso-GL3 in the dried blood spot. The same mutation was detected in her 28-year-old sister who presented with neuropathic pain, hypohidrosis, cornea verticillata, proteinuria (up to $3 \mathrm{~g} / 24 \mathrm{~h}$ ), and typical "zebra bodies" in the renal biopsy.

The prevalence of Fabry disease was $0.53 \%$ (95\% CI $0.29-0.78)$ in males and $0.05 \%$ (95\% CI $0-0.16)$ in females. In males, Fabry disease was more prevalent in the 40-49 years age group and less common in the 1829 years and $50-59$ years age groups (Fig. 1). There were no cases of Fabry disease in males over 60 years of age. 


\section{Mutations in the GLA Gene}

Seventeen different mutations in the GLA gene were identified in 20 patients, including 12 missense mutations and 5 nonsense mutations (Table 1). There were 5 novel mutations (c.400T>G, c.786delG, c.508G $>$ T, c.1197G $>$ A, c. $166 \mathrm{~T}>\mathrm{A}$ ) that were pathogenic, according to the computer analysis (Alamut ${ }^{\circledR}$ Visual). All 5 patients fulfilled the criteria for a definite diagnosis of Fabry disease [8]. One missense mutation ( $\mathrm{p} .161 \mathrm{~T}>\mathrm{C}$ ) was found in 3 patients, including 2 brothers, and one nonsense mutation (c.658C $>$ T) was present in 2 patients. c.786delG mutation leads to the termination of translation and does not shift the reading frame. Therefore, it was attributed to nonsense mutations. One patient (\#3) with a 13-year history of kidney disease (proteinuria up to $3.2 \mathrm{~g} /$ day and progressive decline in kidney function, hemodialysis for the last 6 months) and no classic manifestations of FD had a late onset phenotype mutation with predominantly cardiac involvement (N215S). Kidney biopsy was unsuccessful. He showed increased Lyso-GL3 level, reduced AGAL activity, mild left ventricular hypertrophy $(13 \mathrm{~mm})$, and white matter lesions on MRI. No patients with the same mutation on dialysis have been described yet in the literature.

\section{Course of CKD}

The duration of renal replacement therapy (RRT) at the time of screening ranged from 0 to 13 years (median of 4 years) and exceeded 3 years in 13 patients $(65.0 \%)$. Three patients underwent successful kidney transplantation.

First signs of kidney disease were found at the age of 15-57 years (median of 34 years). In 7 patients, exact proteinuria prior to the development of ESRD was unknown. Thirteen patients presented with a history of mild-to-moderate proteinuria $(0.4-5.0 \mathrm{~g} / 24 \mathrm{~h}$, median of $2.0 \mathrm{~g} / 24 \mathrm{~h}$ ) without hematuria or leukocyturia. Proteinuria was less than $2 \mathrm{~g}$ in 9 of 13 patients (69.2\%). Only 1 patient developed the nephrotic range proteinuria $(5.0 \mathrm{~g} / 24 \mathrm{~h})$ that was not associated with an overt nephrotic syndrome. Only 2 patients underwent kidney biopsy prior to RRT (unsuccessful in one them). In 1 patient, glomerulosclerosis was found. FD was not suspected. There were different reasons explaining why biopsy was not performed in the other patients: advanced $\mathrm{CKD}$ at the first presentation, poor quality of medical care in the small cities, patients did not seek consultation of the nephrologist, and so on.

Six patients (30.0\%) had ESRD at the first presentation, while in the other patients RRT was initiated within 3-13 years (median of 4 years) after detection of protein-

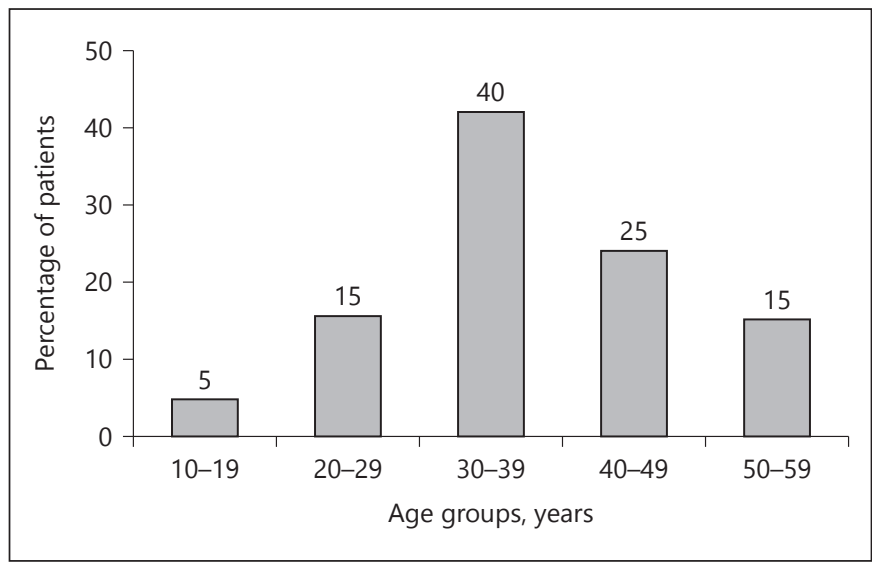

Fig. 2. Distribution of age of patients at the initiation of dialysis.

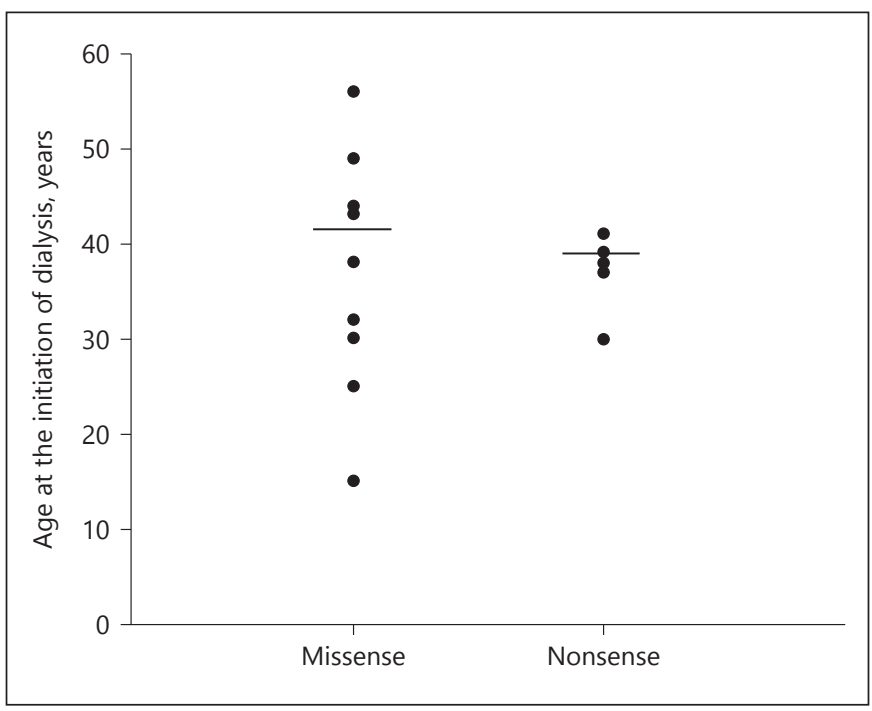

Fig. 3. Age at the initiation of dialysis in patients with missense and nonsense mutations.

uria. Age of patients at the start of dialysis ranged from 15 to 56 years (median of 40 years). The majority of patients started RRT at the age of 20-49 years (Fig. 2). The median age of patients with missense and nonsense mutations was similar at the initiation of dialysis (41 and 39 years, respectively; Fig. 3). We did not compare Lyso-GL3 levels in patients with different types of mutations due to a low number of patients.

\section{Clinical Features of Fabry Disease in Dialysis Patients}

Sixteen of 20 patients (80.0\%) had a history of "classic" symptoms of Fabry disease from childhood or adolescence, including neuropathic pain in 16 patients $(80.0 \%)$, 
angiokeratoma in 7 patients (35.0\%), and hypohidrosis or anhidrosis in 16 patients (80.0\%). However, diagnosis of Fabry disease was never considered.

All 20 patients had left ventricular hypertrophy on echocardiography (maximal wall thickness in diastole $>12 \mathrm{~mm}$ ). The presence of late gadolinium enhancement in cardiac magnetic resonance as an indicator of myocardial fibrosis was detected in 4 (44.4\%) of 9 patients. Eight of 20 patients (40.0\%) had a history of ischemic stroke. Brain MRI showed white matter lesions in 11 of 17 patients (64.7\%).

Fourteen of 20 patients with identified Fabry disease started enzyme replacement therapy; of which two patients $(10.0 \%)$ have died (recurrent stroke in 1 patient and sudden cardiac death in the other patient).

\section{Discussion}

In the Russian nationwide screening program, the prevalence of Fabry disease in 5,572 dialysis patients was $0.36 \%$. As expected, in males it was 10 -fold higher than in females (0.53 and $0.05 \%$, respectively). In a recent metaanalysis of 20 screening studies in dialysis units, the average prevalence of Fabry disease was similar to our data and ranged from $0.10 \%$ in females to $0.33 \%$ in males [10]. In 2015 , there were approximately 35,000 patients treated with hemodialysis in the Russian Federation. Therefore, the actual number of patients with FD and ESRD probably exceeds $120-130$.

The majority of our patients with Fabry disease (80.0\%) started RRT at the age of 20-49 years. The other authors reported similar data. In the United States Renal Disease System database, the mean age of Fabry patients who initiated dialysis was 42 years [5]. Our data suggest that the highest prevalence of Fabry disease in dialysis units (up to $1: 100$ ) can be expected in males at the age of $30-$ 49 years. According to the recommendations by the European Renal Best Practice, patients should be screened for Fabry disease when there is unexplained CKD in males younger than 50 years and females of any age [11].

Over $80 \%$ of dialysis patients with Fabry disease that was established by screening in our study had a history of specific symptoms including neuropathic pain, angiokeratoma, and/or hypohidrosis/anhidrosis. However, diagnosis of Fabry disease was never considered even in patients with overt clinical features who had affected relatives with similar manifestations. The median time from the symptoms onset to the initiation of dialysis was 23 years (up to 40 years). A higher awareness of Fabry disease among nephrologists is apparently essential for

Prevalence and Clinical Features of Fabry Disease in Hemodialysis Patients timely diagnosis. Notably, data from the Fabry Outcome Survey showed that over the last decade the delay in diagnosis of Fabry disease did not improve, despite a trend towards earlier diagnosis in adults and children [12]. Renal biopsy is important for establishing diagnosis of Fabry nephropathy and exclusion of other kidney diseases, particularly chronic tubulointerstitial nephritis caused by a prolonged use of analgesics in patients with neuropathic pain.

In our study, the type of mutation in the GLA gene did not predict a more rapid progression of CKD in patients with Fabry disease, since the median age of patients with missense and nonsense mutations was almost identical at the initiation of dialysis. However, the lack of difference may be due to a small sample size. Only 2 mutations were present in more than 1 patient. c.161T $>C$ mutation in the GLA gene was found in 3 dialysis patients, including 2 brothers at the age of 47 and 48 years. Their mother and grandfather were also treated with hemodialysis and died at the age of 51 and 57 years, respectively. It seems that certain mutations may be associated with the renal phenotype of Fabry disease.

Nephropathy in Fabry disease initially manifests by albuminuria that progress to overt proteinuria. In a cross-sectional retrospective analysis of 1,262 adult patients from the Fabry Registry, the nephrotic range proteinuria was a rare finding $(2-3 \%)$ in patients with CKD stage 1 or 2 but its prevalence increased steeply up to $18-27 \%$ in advanced CKD stages [4]. However, our patients presented with a history of mild-to-moderate proteinuria (median of $2.0 \mathrm{~g} / 24 \mathrm{~h}$ ) that was not associated with an elevated blood pressure, usually did not increase with time and reached the nephrotic range in only $1 \mathrm{pa}-$ tient. Arterial hypertension developed only in advanced CKD stages.

Proteinuria is an established factor associated with progressive loss of kidney function in patients with Fabry disease. Schiffman et al. [13] studied the natural course of Fabry disease in 447 patients. The mean rates of estimated glomerular filtration rate decline for males with baseline proteinuria $<0.1 \mathrm{~g} / 24 \mathrm{~h}, 0.1-1 \mathrm{~g} / 24 \mathrm{~h}$, and $\geq 1 \mathrm{~g} / 24 \mathrm{~h}$ were $-1.6,-3.3$, and $-6.9 \mathrm{~mL} / \mathrm{min} / 1.73 \mathrm{~m}^{2} /$ year, respectively. Therefore, patients with overt proteinuria, particularly higher than $1 \mathrm{~g} / 24 \mathrm{~h}$, can rapidly reach ESRD within a few years. In our cohort, RRT was initiated within 3-13 years (median of 4 years) after detection of proteinuria.

The age of dialysis patients with Fabry disease detected by screening in our study ranged from 28 to 58 years. Untreated patients in this age group show accumulation of glycosphingolipids in different tissues and organs, in- 
cluding heart and brain. Left ventricular hypertrophy of different degree was found in all patients and was frequently associated with myocardial fibrosis on late gadolinium-enhanced cardiac magnetic resonance. Over 60\% of patients presented with white matter lesions on brain MRI, and $40 \%$ of patients had a history of stroke. Two patients died: one of them suffered a recurrent stroke and the other experienced a sudden cardiac arrest. However, the duration of RRT was relatively long (median of 4 years) and exceeded 5 years (up to 13 years) in one third of patients. Data from 2,712 patients in the Fabry Registry showed that male and female patients who received RRT experienced more cardiovascular events and strokes than did other patients. A total of 92 of 186 males who were treated with RRT (50\%) had a history of a cardiac event or stroke, compared to 230 of 1,173 non-RRT males (20\%) [14]. This finding was expected given a well-known contribution of advanced CKD to an increased risk of major cardiovascular events and death.

Our study had limitations. We measured AGAL activity for screening in both males and females. However, enzyme expression can be within the normal range in up to $60 \%$ of heterozygous females [15]. Therefore, the prevalence of Fabry disease in female patients undergoing RRT was underestimated, since sequencing of the GLA gene is necessary for the diagnosis in most females [6]. We also lack positive renal pathology, and Lyso-GL-3 was not tested in 9 of 20 patients. Nevertheless, we evaluated the prevalence of Fabry disease in one of the largest cohort of patients undergoing dialysis in the different regions of Russia.

\section{Conclusion}

Our study showed a low awareness of Fabry disease among the Russian nephrologists, given the presence of classical variant of disease in the majority of patients who were detected by screening. Therefore, wide-spread screening in at-risk patients remains the feasible approach to diagnose Fabry disease in patients with ESRD and their family members, particularly in patients with late-onset or attenuated phenotype who lack early alerting symptoms. The highest prevalence of Fabry disease in dialysis units can be expected in males in the age group of $30-50$ years. AGAL activity is an inappropriate parameter for screening in female patients with ESRD and should be replaced by more reliable markers.

\section{Acknowledgments}

The authors wish to acknowledge all patients and physicians who participated in the screening program.

The results presented in this paper have not been published previously in whole or part, except in the abstract form.

\section{Ethics Statement}

The authors have no ethical conflicts to disclose.

\section{Disclosure Statement}

The authors acknowledge the following potential conflicts of interest: S.M. received travel grants and honoraria for lectures and serving on advisory boards from Shire and Sanofi Genzyme. V.F. received travel grants and honoraria for lectures and serving on advisory boards from Sanofi Genzyme. L.N.-B., K.S., and A.P. received travel grants from Shire. A.M., A.S. have no competing interests to declare.

\section{Funding Sources}

Funding for laboratory testing was provided by Shire. The employees of Shire did not participate in the analysis or interpretation of data and in the preparation of manuscript. The work was supported by the "Russian Academic Excellence Project 5-100".

\section{References}

1 Germain DP: Fabry disease. Orphanet J Rare Dis 2010;5:30.

2 Echevarria L, Benistan K, Toussaint A, et al: $\mathrm{X}$-chromosome inactivation in female patients with Fabry disease. Clin Genet 2016:89: 44-54.

3 Wilcox WR, Oliveira JP, Hopkin RJ, et al: Females with Fabry disease frequently have major organ involvement: lessons from the Fabry Registry. Mol Genet Metab 2008;93:112-128.
4 Ortiz A, Oliveira JP, Waldek S, et al; Fabry Registry: Nephropathy in males and females with Fabry disease: cross-sectional description of patients before treatment with enzyme replacement therapy. Nephrol Dial Transplant 2008;23:1600-1607.

5 Thadhani R, Wolf M, West ML, et al: Patients with Fabry disease on dialysis in the United States. Kidney Int 2002;61:249255.
6 Eng CM, Fletcher J, Wilcox WR, et al: Fabry disease: baseline medical characteristics of a cohort of 1765 males and females in the Fabry Registry. J Inherit Metab Dis 2007;30:184-192.

7 Schiffmann R, Hughes DA, Linthorst GE, et al: Screening, diagnosis, and management of patients with Fabry disease: conclusions from a "kidney disease: improving global outcomes" (KDIGO) controversies conference. Kidney Int 2017;91:284-293. 
8 Namazova-Baranova LS, Baranov AA, Pushkov AA, Savostyanov KV: Fabry disease in children: a federal screening programme in Russia. Eur J Pediatr 2017;176:13851391.

9 Smid BE, Van der Tol L, Cecchi F, et al: Uncertain diagnosis of Fabry disease: consensus recommendation on diagnosis in adults with left ventricular hypertrophy and genetic variants of unknown significance. Int J Cardiol 2014;177:400-408.

10 Linthorst G, Bouwman M, Wijburg F, et al: Screening for Fabry disease in high-risk pop- ulations: a systematic review. J Med Genet 2010;47:217-222

11 Terryn W, Cochat P, Froissart R, et al: Fabry nephropathy: indications for screening and guidance for diagnosis and treatment by the European Renal Best Practice. Nephrol Dial Transplant 2013;28:505-517.

12 Reisin R, Perrin A, García-Pavía P: Time delays in the diagnosis and treatment of Fabry disease. Int J Clin Pract 2017;71.

13 Schiffmann R, Warnock DG, Banikazemi M, et al: Fabry disease: progression of nephropathy, and prevalence of cardiac and cerebro- vascular events before enzyme replacement therapy. Nephrol Dial Transplant 2009;24: 2102-2111.

14 Ortiz A, Cianciaruso B, Cizmarik M, et al: Endstage renal disease in patients with Fabry disease: natural history data from the Fabry Registry. Nephrol Dial Transplant 2010;25:769-775.

15 Gupta S, Ries M, Kotsopoulos S, et al: The relationship of vascular glycolipid storage to clinical manifestations of Fabry disease: a cross-sectional study of a large cohort of clinically affected heterozygous women. Medicine (Baltimore) 2005;84:261-268. 